Rosa Ma Jiménez Catalán

Universidad de La Rioja

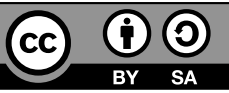

\title{
Asociaciones prototípicas en la producción y disponibilidad léxica de adolescentes españoles y eslovenos en español y en inglés como lenguas extranjeras
}

Palabras clave: prototipos, asociaciones de palabras, vocabulario productivo, disponibilidad léxica, partes del cuerpo, la ropa, lengua extranjera, español, inglés

\section{Introducción}

Nuestro artículo compara el léxico disponible en inglés y en español como lenguas extranjeras (LE) a partir de dos centros de interés o categorías semánticas: Partes del cuerpo (Parts of the body) y La ropa (Clothes). En primer lugar, identificamos el número de palabras producidas en inglés por adolescentes españoles para luego comparar dicha producción con la de adolescentes eslovenos aprendientes de español LE. En segundo lugar, identificamos las tres palabras más disponibles en inglés y las comparamos a las tres más disponibles en español. Los prototipos semánticos permiten comprender la manera en que un aprendiente categoriza una LE. Su estudio en dos lenguas extranjeras, provenientes de adolescentes en dos países, nos permite abordar las asociaciones prototípicas en un marco más amplio. Si los resultados son similares, nuestro estudio aportará evidencia de la universalidad de los prototipos en las categorías semánticas analizadas. Por el contrario, de observarse diferencias, los resultados aportarán datos de la existencia de rasgos culturales específicos a cada lengua. 
En primer lugar, presentamos el marco teórico y empírico de nuestra investigación, repasando las principales teorías sobre prototipos, así como los estudios que se han realizado sobre este tema en LE. En segundo lugar, describimos el diseño, la muestra, el instrumento de recogida de datos y los procedimientos de edición, lematización y análisis. Seguidamente, presentamos los resultados obtenidos por los aprendientes de inglés y procedemos a su cotejo con los resultados de los aprendientes de español, acompañados de su interpretación a la luz de la teoría y la investigación previa sobre prototipos en LE. Finalmente, presentamos nuestras conclusiones y sus implicaciones tanto para los estudios de léxico disponible como para la pedagogía de vocabulario del español y del inglés como LE.

\section{Marco Teórico y Empírico}

\subsection{Teorías de prototipos}

La teoría clásica de la categorización aristotélica basada en las condiciones necesarias y suficientes cambia considerablemente debido a la noción de prototipo propuesta por Rosch (1975). Esta investigadora denomina prototipo al modelo que es mejor, central y más representativo en una categoría. Es decir que en la categoría fruta, el mejor ejemplo es la manzana; y el miembro poco representativo es la aceituna. Así, el prototipo implica ser el ejemplar mejor reconocido en una categoría por el ser humano. Actúa como punto de referencia cognitivo en el que se considera que los miembros prototípicos son los que primero se adquieren en la infancia, los que se categorizan más rápidamente y los que aparecen en primer lugar en un ejercicio de enumeración de los miembros de una categoría. El concepto de prototipo amplía su alcance en la categorización bajo diferentes modelos. Por ejemplo, según Murphy (2002) los miembros de una categoría se clasifican en miembros muy típicos o buenos, o en miembros menos típicos o menos buenos. Por su parte, Dubois (1982, 1983 en Kleiber, 1995) reveló que el prototipo es el mejor ejemplar cuando es el elemento más utilizado, en lugar de ser el caso central de la categoría únicamente. Kleiber confirmó las características de los prototipos en el proceso de categorización y afirmó que el significado léxico de una palabra encuentra su fundamento en el parecido existente con el caso central, el prototipo. Para ahondar un poco más en la definición de prototipo y su teoría, se exploran dos versiones "la versión estándar" y "la versión ampliada". En la primera, el prototipo, además de ser el ejemplar idóneo definido por la frecuencia de su uso para establecer su pertenencia a una categoría, es introducido bajo nuevos factores 
de organización. Por una parte, encontramos la estructuración interna de las categorías (Dimensión Horizontal) y por otra, las grandes líneas de la estructuración intercategorial (Dimensión Vertical). La dimensión horizontal establece la organización jerárquica de la categoría en tres niveles (superordinado, básico y subordinado). Es decir que la categoría animal, por ejemplo, estará localizada en el nivel superordinado, puesto que este nivel agrupa ejemplares que no están representados por una imagen mental. En el nivel básico encontramos la palabra perro por ser el nivel más inclusivo en el que los miembros de una categoría poseen un gran número de atributos comunes. Y, finalmente, en el nivel subordinado, se ubica la palabra boxer por ser una subclase que aporta información adicional sobre los detalles específicos de un objeto, en este caso, una clase de perro. El nivel básico se convierte en el eje principal de la dimensión vertical por ser el nivel más informativo y de identificación más rápida con mayor número de atributos comunes (Kleiber, 1995). Sin embargo, no todos los casos pueden ser considerados prototípicos y por tal motivo, se genera un problema de pertenencia a una categoría. Para Rosch y Lloyd (1973), el prototipo deja de ser el ejemplar idóneo de una categoría para convertirse en efectos prototípicos, también llamados modelos cognitivos idealizados (Lakoff, 1987). Estos modelos son elementos cuya pertinencia se ejerce en múltiples tareas cognitivas, principalmente lingüísticas, en las cuales se pasa de una concepción monosémica, como la representada en la versión estándar, a una concepción inicialmente basada en la semejanza de familia. Esta concepción permite que los miembros de una categoría puedan estar ligados unos a otros en una relación polisémica sin que exista una propiedad común que defina la categoría. Es decir, como afirma Lakoff, el fenómeno de la polisemia aparece como un caso especial de la categorización con base prototípica, en donde los sentidos de una palabra son los miembros de una categoría. Ambas versiones aportan de manera distinta la forma de comprender la teoría del prototipo en la categorización y las ventajas arrojadas en cada una de sus interpretaciones permiten desarrollar estudios sobre casos distintos de categorías semánticas y lingüísticas. En cuanto a la relación entre las teorías de los prototipos y la disponibilidad léxica, resaltamos la idea de grado de disponibilidad (Dimitrijević,1969; Samper Hernández, 2002; Hernández Muñoz, 2006; Šifrar Kalan, 2009, 2016; Bartol, 2010; Paredes García, 2015; Ávila Muñoz, 2017). Es decir, cuanto mayor sea recordada y enunciada una palabra por un grupo de informantes, mayor será el grado de disponibilidad y mayor su prototipicidad. De esta manera, el grado de disponibilidad de una unidad léxica será evidenciado por los miembros más típicos o menos típicos del prototipo dentro de una categoría semántica. 
En nuestro estudio, específicamente analizaremos dos categorías semánticas con grupos de informantes adolescentes. Describiremos entonces, algunos postulados sobre esta etapa como desarrollo de abstracción, categorización y de prototipos.

\subsection{Etapa adolescente}

El desarrollo cognitivo, según Piaget e Inhelder (1969) es un proceso que no solo consiste en lo que el individuo conoce, sino como organiza el conocimiento para razonar sobre su mundo. Por tal motivo, Piaget desarrolló una secuencia de cuatro etapas, donde cada una de ellas muestra una variación individual y de grupo. En cuanto a los adolescentes, objeto de nuestro análisis, se ubican en la etapa de operaciones formales, la cual se caracteriza por la expansión del pensamiento más allá de lo concreto. De esta manera, como señala Flavell (1985), los adolescentes desarrollan sistemas abstractos basados en la lógica y el razonamiento que les permite reflexionar sobre sus procesos de pensamiento, discutir problemas complejos sobre ideas abstractas, hacer predicciones sobre hechos hipotéticos, realizar operaciones mentales, usar sistemas de símbolos y definir conceptos a través de la categorización. Además de estos postulados de la escuela Piagetiana, es necesario contemplar la caracterización que hace (Vigotsky, 1995) sobre el lenguaje. Para este autor el lenguaje es la herramienta psicológica que más influye en el desarrollo cognitivo, puesto que proporciona el medio para expresar ideas y formular preguntas, así como establecer categorías y definir conceptos.

Partiendo de los postulados mencionados sobre el desarrollo cognitivo de los adolescentes, analizaremos la categorización de dos categorías semánticas en español e inglés como LE: Partes del cuerpo (Parts of the body), La ropa (Clothes). Como señala Scalozub (2007), el cuerpo tiene un gran protagonismo en la adolescencia; mediante el cual se transmiten marcas de identidad y pertenencia a una cultura de grupo como, por ejemplo, los tatuajes en la piel, o el piercing en distintas partes del cuerpo. Para hacer frente a estos cambios de identificación, el adolescente hará uso de su cuerpo como un contenedor de las transformaciones y como representante de su experiencia para poder comunicarse con su entorno (Orduz, 2010). La ropa, por su parte, representa los modelos a quienes se quieren parecer los adolescentes, quienes, mediante la ropa, los adornos o el modo de peinarse reflejan las características de: “...un determinado grupo ya sea con referencia a un estilo musical (Rockeros, Punkis, Mods, Tecno, Heavy, Rastas, Raperos etc.) o bandas de 
distinta adscripción política o simplemente asociaciones para defensa territorial al margen de la ley (Maras, Neonazis etc.)” (Iglesias 2015:59).

\section{Revisión de estudios: prototipos en lenguas extranjeras}

Durante las últimas décadas, se han realizado estudios sobre prototipos tanto en lenguas nativas como extranjeras. En este caso, aproximadamente ocho de las investigaciones revisadas se centran en lenguas diferentes como: inglés, español, chino, suajili (Tanzania), esloveno, polaco, alemán, cantonés, turco y finlandés. Centrándonos en el inglés como LE, en los estudios de Ibbotson y Tomasello (2009) así como Supasiraprapa (2017), los prototipos fueron presentados a través de construcciones en la adquisición temprana de una lengua y los efectos prototípicos en aprendientes de una primera y una segunda lengua en la adultez. El primer estudio con niños alemanes, polacos, cantoneses e ingleses, el segundo con adultos chinos e ingleses arrojaron resultados de prototipicidad en cuanto al tipo de producciones orales y escritas emitidas por cada uno de los grupos tanto en inglés como lengua nativa como en lengua extranjera a través del uso de oraciones transitivas del patrón de (SVO) sujeto-verbo-objeto. La comparación señaló que, para los hablantes de inglés nativo, seguir este tipo de patrón transitivo les tomaba más tiempo que al grupo de hablantes de inglés L2, debido al hecho que este proceso se hace de forma natural. Por otra parte, investigaciones como las de Yuan (1990) y Lukusa (1996) revelaron nociones prototípicas con informantes chinos y tanzanos adultos y adolescentes haciendo uso de los adjetivos delgado (thin) y responsable (responsible) en inglés como LE. Los resultados obtenidos indicaron las diferencias culturales y lingüísticas que tienen estos dos adjetivos para cada grupo de hablantes, tanto en su lengua nativa como en el aprendizaje de una segunda lengua (inglés). En cuanto a estudios de disponibilidad léxica, específicamente en español como L1, se comparan diferentes regiones dialectales (Alba, 1998; Alba, 2000; López Chávez, 1995; Samper Padilla, 1999) y se muestra que, a pesar de las diferencias fonéticas y de uso, el léxico demuestra esencialmente características comunes o prototípicas del español que se habla en todo el mundo. Asimismo, los resultados de estudios de disponibilidad léxica tanto en español como en inglés como L2, realizados de forma independiente unos de otros, en países y contextos educativos diferentes, muestran una gran similitud en lo que respecta a las respuestas que los aprendientes aportan a las categorías semánticas utilizadas como estímulos en las pruebas de disponibilidad (véase, Jiménez Catalán, 2014). Finalmente, Šifrar Kalan 
(2016) recogió información de estudiantes preuniversitarios y universitarios eslovenos, finlandeses, españoles, turcos y chinos con objeto de averiguar la existencia o no de la universalidad de los prototipos semánticos en el léxico disponible del español como LE. Los hallazgos revelan una gran similitud en las palabras prototípicas a pesar de las diferencias culturales y de lengua de los informantes. El presente estudio se inspira en este trabajo. En concreto, planteamos averiguar si se observan patrones semejantes en inglés LE que puedan permitir establecer la universalidad de los prototipos en lenguas extranjeras o si, por el contrario, se observan patrones de idiosincrasia cultural. Para ello, adoptamos la metodología de la disponibilidad léxica, entendida esta como las palabras que los individuos tienen en sus mentes y que surgen en respuesta a centros de interés que son válidos para dominios estrechamente relacionados con la vida cotidiana (Jiménez Catalán, 2014).

\section{Metodología}

\subsection{Objetivos}

Nos planteamos, en primer lugar, averiguar si se observan similitudes o diferencias en el número de palabras y vocablos producidos por adolescentes españoles y eslovenos respectivamente en las categorías Parts of the body/Partes del cuerpo, y Clothes/La ropa. Como segundo objetivo, buscamos averiguar si se observan similitudes y/o diferencias en las palabras más disponibles para dichas categorías en inglés y español LE. En concreto, queremos ver si se observa convergencia en los prototipos o si, por el contrario, se constatan divergencias.

\subsection{Diseño}

Este estudio es transversal: se comparan dos muestras de adolescentes provenientes de Eslovenia y España. Comparten un rango de edad y nivel educativo similar, así como el hecho de cursar una lengua extranjera como asignatura en el contexto del aula dentro de sus respectivos centros educativos. Se diferencian en la lengua extranjera objeto de aprendizaje: español en el primer caso, e inglés en el segundo.

\subsection{Muestra}

El número total de informantes es de 200 adolescentes de $2 .^{\circ}$ de bachillerato o equivalente (curso preuniversitario). Se distribuyen en dos grupos, 100 
aprendientes de español como lengua extranjera (Grupo A) y 100 aprendientes de inglés como lengua extranjera (Grupo B). El rango de edad de ambos grupos es 17-18 años. El inglés es una asignatura obligatoria en todos los cursos de educación primaria (6-12 años), secundaria obligatoria (12-16) y bachillerato (16-18). Los datos se recogieron en la primavera de 2015 (marzoabril). En el momento de recogida, los informantes habían acumulado un total de 1500 horas de inglés en el aula. En la selección de las muestras seguimos dos procedimientos diferentes. Para el Grupo A tomamos como referencia las investigaciones de Šifrar Kalan $(2009,2016)$ sobre léxico disponible y prototipos de adolescentes preuniversitarios provenientes de cuatro centros de Eslovenia. Para los estudiantes eslovenos, el español es una asignatura optativa con un nivel aproximado de B1, de acuerdo con el Marco Común Europeo de Referencia. Los datos se recogieron en la primavera de 2006-2007 y en el momento de la recogida de la encuesta, los informantes habían recibido un total de 300 horas lectivas de ELE (Šifrar Kalan, 2009: 167). Respecto al Grupo B, nos basamos en una selección aleatoria a partir de una muestra representativa del alumnado adolescente en $2 .^{\circ}$ de bachillerato en seis institutos de educación secundaria públicos de La Rioja (España), comunidad autónoma con competencias educativas propias. Los datos se recogieron mediante técnica estratificada por miembros del grupo de investigación de lingüística aplicada de la Universidad de La Rioja (GLAUR), dentro del marco de un proyecto nacional de investigación sobre léxico disponible de aprendientes de inglés como lengua extranjera (Ref. FFI 2013-47707-P). En este artículo, extendemos la investigación al análisis de la producción de asociaciones prototípicas, dentro del marco de nuestro nuevo proyecto nacional de investigación, INVOPLEX, (Ref. PG2018-095260-B-100).

\subsection{Instrumento de recogida de datos}

En ambos grupos los datos se recogieron mediante una encuesta de disponibilidad léxica compuesta de dos partes. En la primera, se incluyeron preguntas destinadas a obtener información sobre el perfil lingüístico del aprendiente como edad, curso, sexo/género, lengua materna, y conocimiento de otras lenguas. En el caso del Grupo B, se incluyeron también preguntas encauzadas a obtener información sobre estancias en países de habla inglesa, asistencias a cursos de verano, o clases de inglés particulares externas al centro educativo. En la segunda parte y para ambos grupos, se incluyeron centros de interés o categorías semánticas destinadas a estimular la activación y producción de 
palabras en una prueba escrita controlada. En la misma, se pidió a los aprendientes que escribieran cuantas palabras le viniesen a la mente relacionadas con el centro de interés. La medida fue la producción de palabras diferentes o vocablos en dos minutos por cada centro de interés o categoría semántica. En el presente estudio nos centraremos en la comparación de patrones prototípicos en dos centros de interés: Partes del cuerpo (Parts of the body), La ropa (Clothes). Las razones que justifican esta selección se basan por una parte en la limitación de espacio para realizar un análisis detallado de todos los centros de interés, pero fundamentalmente, en la relación de los dos primeros con los temas que preocupan en la etapa adolescente como son el cuerpo o la ropa.

\subsection{Procedimientos}

Una vez obtenidos los correspondientes permisos de recogida de datos en los institutos de secundaria, se administraron las encuestas en el aula de los informantes y seguidamente se procedió a la codificación, edición y lematización de los datos. Todas las encuestas fueron codificadas asignando un número de identificación a cada informante. Se codificaron también las variables edad, sexo y centro educativo, así como el número total de palabras y vocablos vertidos en la prueba y por centro de interés. Seguidamente se procedió a la edición y lematización de todas las palabras vertidas en las encuestas.

Siguiendo los procedimientos de nuestras investigaciones previas (Jiménez Catalán, 2014; Jiménez Catalán y Agustín Llach, 2017; Jiménez Catalán y Fernández Fontecha, 2019), en el presente trabajo adoptamos la unidad léxica de significado como medida de análisis. De esta manera, englobamos tanto las palabras simples (kitchen) y compuestas (bedroom) como combinaciones de palabras que poseen significado en su conjunto como, por ejemplo, combinaciones de verbos y preposiciones o adverbios (give up, put up with) así como las frases idiomáticas (flying bonours). En cuanto a las normas de edición, seguimos las establecidas en nuestros anteriores trabajos de léxico disponible, las cuales coinciden en gran medida con las adoptadas en los estudios de español como L2 (Carcedo González, 2000; Samper Hernández, 2002) y aplicadas por Šifrar Kalan (2009, 2016).

\section{Resultados y discusión}

Respecto a nuestro primer objetivo, nos propusimos averiguar si se observan similitudes o diferencias en el número de palabras y vocablos producidos 
por adolescentes españoles y eslovenos aprendientes de inglés y de español como LE respectivamente en las categorías Parts of the body/Partes del cuerpo y Clothes/La ropa. La Tabla 1 muestra los resultados totales y las medias para cada categoría. Observamos que los adolescentes españoles activaron un mayor número de palabras en la categoría Parts of the body, pero un número mayor de vocablos o palabras diferentes en Clothes. Los datos de las medias se obtuvieron a partir de las medias individuales de vocablos vertidos en respuesta a cada categoría por cada estudiante. Esta medida nos indica que la producción de vocablos en inglés de la muestra de adolescentes españoles es mayor en el campo semántico Parts of the body que en Clothes. Sin embargo, es importante precisar que tan solo el $51,3 \%$ alcanzó la media en la primera categoría y un $47,1 \%$ en la segunda. Los datos de la desviación típica corroboran esta tendencia, la cual es similar en ambas categorías.

\begin{tabular}{|l|c|c|c|c|}
\cline { 2 - 5 } \multicolumn{1}{c|}{} & Palabras & Vocablos & $\begin{array}{c}\text { Media } \\
\text { vocablos }\end{array}$ & Desv. Típica \\
\hline Parts of the body & 1582 & 120 & 15,67 & 5,46 \\
\hline Clothes & 1326 & 143 & 13,26 & 6,06 \\
\hline
\end{tabular}

Tabla 1. Descriptivo de la producción léxica por categorías semánticas en inglés LE

Los resultados para las categorías analizadas son semejantes a los obtenidos en Polonia (López González, 2014) y en Eslovenia ( Šifrar Kalan, 2016) con aprendientes de español como LE, en los cuales 'Partes del cuerpo' concentró un número mayor de vocablos que 'La ropa'. Sin embargo, en lo que respecta a la comparación de medias, nuestros resultados presentan una mayor convergencia con los obtenidos por López González (2014) con adolescentes polacos aprendientes de español en secundaria, quienes produjeron una media de 15,52 palabras para la categoría 'Partes del cuerpo' y 12,02 palabras para la categoría 'La ropa'. Por el contrario, nuestros resultados divergen de las medias obtenidas por los adolescentes eslovenos, quienes de acuerdo con el aporte de Šifrar Kalan (2016) produjeron respectivamente 12,9 y 9,86 vocablos. Estas diferencias pueden deberse a variables propias de cada contexto educativo como, por ejemplo, el número de horas de instrucción formal recibidas por ambos grupos de informantes. Estas diferencias son notorias puesto que en el momento de recogida de datos los adolescentes eslovenos habían recibido 300 horas de español en el aula, como segunda lengua optativa (Šifrar Kalan 
2009: 167). En comparación, en España el inglés es una asignatura obligatoria en el currículum desde el primer curso de Educación Primaria (6 años) hasta el último curso de Educación Secundaria Obligatoria (ESO) (16 años), así como en los dos años de bachillerato (Educación Secundaria no obligatoria) (17-18 años). Al final del segundo curso de bachillerato, los aprendientes de inglés acumulan aproximadamente 1500 horas de instrucción en el aula. Por consiguiente, si comparamos las medias desde la perspectiva del total de horas recibidas de instrucción de la lengua extranjera, los adolescentes eslovenos obtienen mejores resultados, al menos en lo que respecta a los dos campos semánticos analizados.

En nuestro segundo objetivo nos propusimos averiguar si se dan similitudes y/o diferencias en las palabras más disponibles de los adolescentes españoles en inglés y eslovenos en español, respectivamente, lenguas extranjeras en sus correspondientes currículos. La Tabla 2 muestra los índices de disponibilidad de las tres palabras más disponibles para ambos grupos, para las cuales disponemos de índice de disponibilidad del grupo esloveno. Los datos señalan que para los adolescentes españoles eye, nose, y bead son las tres más disponibles en la categoría Parts of the body, con 95 informantes (de 100), 90 (de 100) y 72 (de 100) respectivamente que produjeron dichas respuestas. Mientras que para el grupo de adolescentes eslovenos cabeza, mano y ojo son las tres más disponibles. No obstante, encontramos divergencia en las posiciones de los índices de las tres primeras respuestas de ambos grupos en la categoría Clothes: mientras que $t$-sbirt es la palabra más disponible en inglés, seguida de trousers y jeans, en español las tres más disponibles son pantalón, camisa y zapato.

\begin{tabular}{|l|l|l|}
\hline Cl & España & Eslovenia \\
\hline Parts of the body/Partes del Cuerpo & 1. Eye $(0,69)$ & 1. Cabeza $(0,73)$ \\
& 2. Nose $(0,60)$ & 2. Mano $(0,70)$ \\
& 3. Head $(0,58)$ & 3. Ojo $(0,57)$ \\
\hline Clothes/La ropa & 1.T-Shirt $(0,81)$ & 1. Pantalón $(0,67)$ \\
& 2. Trousers $(0,60)$ & 2. Camisa $(0,61)$ \\
& 3. Jeans $(0,54)$ & 3. Zapato $(0,40)$ \\
\hline
\end{tabular}

Tabla 2. Índice de las tres palabras más disponibles en inglés y español LE

La tendencia observada en las tres palabras más disponibles se constata en la comparación de las posiciones de las diez más disponibles en ambos grupos (Tabla 3). Respecto a las respuestas evocadas para Parts of the body/Partes del Cuerpo, los datos muestran ocho palabras en común, si bien las posiciones en 
las respectivas listas difieren. En cuanto a Clothes /La ropa, los datos indican convergencia en siete de las diez palabras más disponibles de los dos grupos. Para los aprendientes de inglés, el porcentaje de informantes que produjeron la palabra como respuesta a Parts of the body fue entre el $70 \%$ y el $100 \%$ de la muestra, en concreto el número de adolescentes españoles aprendientes de inglés que produjeron cada una de las diez palabras se incluye entre paréntesis: Eye (95), Nose (90), Arm (85), Leg (82), Finger (78), Hair (76), Mouth (75), Head 72), Hand (71), Ear (70). En cuanto a Clothes, la distribución de las diez palabras por número de informantes fue la siguiente: T-shirt (96), Trousers (88), Jeans (86), Shoe (83), Jacket (53), Shirt (53), Skirt (49), Trainers (50), Dress (41), Hat (44).

\begin{tabular}{|c|c|c|}
\hline $\mathrm{Cl}$ & Inglés & Español \\
\hline Parts of the body/Partes del Cuerpo & $\begin{array}{l}\text { 1. Eye } \\
\text { 2. Nose } \\
\text { 3. Head } \\
\text { 4. Arm } \\
\text { 5. Leg } \\
\text { 6. Mouth } \\
\text { 7. Hand } \\
\text { 8. Finger } \\
\text { 9. Hair } \\
\text { 10. Ear }\end{array}$ & $\begin{array}{l}\text { 1. Cabeza } \\
\text { 2. Mano } \\
\text { 3. Ojo } \\
\text { 4. Pierna } \\
\text { 5. Dedo } \\
\text { 6. Nariz } \\
\text { 7. Pelo } \\
\text { 8. Rodilla } \\
\text { 9. Espalda } \\
\text { 10. Boca } \\
\end{array}$ \\
\hline Clothes/La ropa & $\begin{array}{l}\text { 1. T-Shirt } \\
\text { 2. Trousers } \\
\text { 3. Jeans } \\
\text { 4. Shoe } \\
\text { 5. Jacket } \\
\text { 6. Shirt } \\
\text { 7. Skirt } \\
\text { 8. Trainers } \\
\text { 9. Dress } \\
\text { 10. Hat }\end{array}$ & $\begin{array}{l}\text { 1. Pantalón } \\
\text { 2. Camisa } \\
\text { 3. Zapato } \\
\text { 4. Camiseta } \\
\text { 5. Falda } \\
\text { 6. Jersey } \\
\text { 7. Vestido } \\
\text { 8. Vaquero } \\
\text { 9. Corbata } \\
\text { 10. Chaqueta }\end{array}$ \\
\hline
\end{tabular}

Tabla 3. Posición de los diez vocablos más disponibles en inglés y español LE

De la comparación de los datos, deducimos que eye/ojo y bead/cabeza son asociaciones prototípicas compartidas en inglés y en español en la categoría semántica de Parts of the body/Partes del Cuerpo por ser evocadas como primeras respuestas en ambas lenguas. Dichos vocablos, (y en realidad, los diez más disponibles para esta categoría) convergen en ambos grupos y confirman la observación de Hernández Muñoz (2006): se trata de una categoría cerrada 
que evoca respuestas similares y para las cuales el índice de disponibilidad suele ser muy similar en distintos grupos de informantes. Asimismo, nuestros resultados corroboran los postulados de la teoría de Kleiber (1995), en la que los prototipos se corresponden con las palabras de nivel básico de la categoría, se caracterizan por ser más cortas, las más fáciles de recordar y las que primero se aprenden en la infancia. El nivel básico de las diez palabras más disponibles se constata en ambos grupos puesto que todas las respuestas (en inglés y en español) se incluyen en la categoría del cuerpo humano, el cual, como señalan Gerstl y Pribbenow (1995), se conceptualiza como un conjunto (el todo) compuesto de un número finito de miembros (las partes). Con base a los resultados se deduce que el cuerpo o body es condición indispensable de Parts of the body $\mathrm{y}$, en consecuencia, se puede entender como nivel supraordinado y las partes como niveles básicos, algunas de las cuales, como señalamos más abajo, se pueden entender como supraordinados que dan lugar a palabras de nivel básico. En nuestra modesta opinión, los datos son relevantes puesto que permiten, por una parte, acceder a la conceptualización del lexicón mental de aprendientes de inglés españoles y aprendientes de español eslovenos y por otra, permiten clasificar las palabras asociadas a Parts of the body/Partes del cuerpo en al menos dos niveles de la jerarquía postulada por Kleiber (1995). Así vemos, que tanto en inglés como en español las primeras palabras evocadas señalan la parte superior del cuerpo humano como la cabeza, los ojos, la nariz, la boca, o el pelo y solo una vez evocadas, los informantes de ambos grupos vierten palabras de las partes central e inferior del cuerpo como arm, hand, finger, leg en inglés o mano, dedo, espalda, pierna, rodilla en el caso del español. Ello sugiere una conceptualización del lexicón mental de los aprendientes de inglés y español similar y en línea con las teorías de (Lakoff y Johnson 1980, 1999; Lakoff y Turner 1989) respecto a la percepción de la experiencia, basada en niveles superiores e inferiores. Desde esta concepción teórica, la cabeza y dentro de ella, los ojos, la nariz, la boca y los oídos se perciben como miembros superiores, siendo la cabeza y los ojos órganos centrales, mientras que las piernas se perciben como miembros inferiores. Si bien todos los miembros son necesarios, algunos son más necesarios que otros; es decir, en ocasiones hemos podido ver seres humanos sin manos o sin piernas, pero no sin cabeza, y es muy difícil imaginar alguien sin ojos. Como señala Paredes García (2015) se trata de una categoría cerrada con base experiencial universal, compartida por todos los seres humanos, dotados de las mismas partes del cuerpo y con atributos similares. Esta concepción está omnipresente en las asociaciones producidas en inglés y español LE de los adolescentes españoles y eslovenos 
y corrobora las comparaciones de español L1 y L2 realizadas por Šifrar Kalan $(2009,2016)$, en las cuales se apuntaba la universalidad de los prototipos al uso del lenguaje por ambas culturas (eslovena y española).

En el caso de Clothes/La ropa, encontramos convergencia, pero también más divergencias que en la anterior categoría analizada. La primera diferencia es que la palabra más disponible para Clothes/Ropa no coincide en español y en inglés, puesto que trousers/pantalón aparece como primera asociación prototípica en español y $t$-shirt en inglés. Esta divergencia también se observa en la lista de los diez vocablos más disponibles en dos sentidos: palabras que coinciden en las dos lenguas, aunque en distinta posición y palabras no coincidentes. Respecto a las primeras: la palabra $t$-sbirt/camiseta en inglés LE, aparece en primer lugar, mientras que en español como LE, se registra en cuarto lugar. La palabra shoe/zapato surge en el cuarto lugar en inglés LE, y en tercer lugar en español como LE. Jeans/vaqueros, por el contrario, dista un poco más en la lista, ya que se ubica en la posición número ocho en las respuestas de los informantes eslovenos y en tercer lugar en las respuestas dadas por el grupo de informantes españoles. Lo anterior nos sugiere que, aunque las posiciones difieren ligeramente, las palabras siguen mostrando evidencia de asociación prototípica en los vocablos más activados por ambos grupos de informantes. Retomamos el planteamiento de Kleiber (1995) arriba señalado en Parts of the body/Partes del Cuerpo para enfatizar las características de producción de palabras prototípicas. A esto añadimos que la categoría semántica de Clotbes/ La ropa es una categoría organizada con una estructura interna de validez universal en torno a un prototipo (Samper Hernández, 2002). De estos postulados, los resultados nos muestran que, a primera vista, en la lista de inglés LE, palabras como: $t$-shirt, jeans y trainers parecen ser prototipos reflejo de la cultura anglosajona. Y, para el caso de la lista de español como LE, las palabras como: vaqueros y jersey, suelen destacar la variante peninsular sobre los anglicismos. Nuestros resultados coinciden con los supuestos observados en los experimentos llevados a cabo por Rosch y Mervis (1975); Larrochelle y Pineau (1994); Warrington y McCarthy (1987) en los que se define a la categoría de Clothes/ La ropa como natural, es decir, una de las más frecuentemente utilizada en experimentos psicolingüísticos, así como en estudios de disponibilidad léxica que sugiere como respuesta a la categoría, palabras de carácter prototípico tales como: trousers/pantalón y shirt/camisa. Observamos que la tendencia de las palabras producidas en ambas categorías se corresponde, como señala Šifrar Kalan $(2009,2016)$, con un alto grado de similitud en las palabras prototípicas a pesar de las culturas y lenguas diferentes de los dos grupos de informantes, 
en este caso (españoles y eslovenos) en la lista de los diez vocablos más disponibles. No obstante, en la interpretación de los resultados se debe tener en cuenta también la presencia de palabras exclusivas a cada lengua en la lista de las diez más disponibles. Así vemos que trainers y bat no están presentes en español, mientras que corbata y jersey no aparecen en inglés. Esto puede ser debido por una parte a aspectos divergentes en la cultura inglesa y española, que pueden haber influido de algún modo en el contenido de los libros de texto, tanto para Parts of the body/Partes del cuerpo como para Clothes/ La ropa, no podemos olvidar que estamos tratando con dos lenguas extranjeras, por consiguiente, estas palabras que vierten ambos grupos han tenido que ser aprendidas a través de la instrucción en clase y del input de los materiales de enseñanza, entre ellos, los libros de texto.

\section{Conclusiones}

En el presente estudio hemos constatado la existencia de asociaciones prototípicas compartidas en inglés y en español como LE en dos categorías Parts of the body/Partes del cuerpo y Clothes/La ropa como bead/cabeza, eye/ojo, $t$-shirt/ camiseta y trousers/pantalón. Estos prototipos quedan patentes en la similitud de palabras respuesta a la lista de los diez vocablos más disponibles, aunque su posición difiere ligeramente en cada grupo. Dichos resultados indican la existencia de un gran número de palabras en común para ambos centros de interés por cada uno de los grupos de informantes en español y en inglés LE. Así, este cotejo arroja resultados de similitud en producción léxica y asociación prototípica, a pesar de tratarse de lenguas y culturas diferentes. No obstante, aunque los resultados señalan similitud, es necesario resaltar que existen variables lingüísticas diferentes en español y en inglés LE en la categoría de Clotbes/ La ropa. Para el grupo de estudiantes eslovenos la variable predominante, es la peninsular (pantalón, camisa, zapato). Mientras que, para el grupo de estudiantes españoles, prevalecen los anglicismos ( $t$-shirt, trousers, jeans).

Respecto a las diferencias de producción léxica entre ambos grupos, en este estudio se reflejan mejores resultados en los estudiantes eslovenos (al menos en los dos campos semánticos analizados). Este resultado es importante a la hora de comparar eslovenos y españoles aprendientes de dos lenguas extranjeras, sin embargo, hemos de ser prudentes en la generalización de los datos puesto que por una parte no se realizaron análisis de estadística inferencial para ver la significancia de las medias, y por otra, las divergencias observadas se pueden deber a variables propias del contexto educativo como, por ejemplo, el enfoque 
metodológico utilizado o el programa de instrucción (intensivo/extensivo), el tipo de input de vocabulario recibido a través de los libros de texto o materiales por cada uno de los grupos de informantes en español y en inglés LE. Los resultados obtenidos aconsejan la conveniencia de llevar a cabo más investigaciones comparativas controlando los posibles factores pedagógicos que pudieran estar afectando la producción de ambos grupos. También, serían necesarios estudios en colaboración para analizar las asociaciones prototípicas e idiosincráticas en inglés y en español de todo el corpus, no únicamente las diez más disponibles y no solo en dos centros de interés, especialmente. La extensión del análisis a todo el corpus nos permitiría averiguar, por ejemplo, si el tipo de asociación se mantiene constante o cambia conforme disminuye el grado de disponibilidad de la palabra. Asimismo, la ampliación de la presente investigación a otros centros de interés nos permitiría conocer si el nivel de la asociación prototípica se mantiene constante independientemente del campo semántico.

\section{Bibliografía}

Alba, O. (1998): «Variable léxica y dialectología hispánica». La Torre, 7-8, 299-316.

Alba, O. (2000): «Variable léxica y comparación dialectal». En: Nuevos aspectos del español en Santo Domingo. Provo/Utah: Brigham Young University, 99-132.

Ávila Muñoz, A.M. (2017): «The available lexicon: A tool for selecting appropriate vocabulary to teach a foreign language». Iranian Journal of Language Teaching Research, 5(1), 71-91.

Bartol Hernández. J.A. (2010): «Disponibilidad léxica y selección del vocabulario». En: Rosa María Castañer-Martín, Vicente Lagüéns-Gracia (coords.), De moneda nunca usada. Estudios dedicados a José María Enguita Utrilla. Zaragoza: Instituto Fernando el Católico, 85-107.

Carcedo González, A. (2000): Disponibilidad léxica en español como lengua extranjera: el caso finlandés (estudio del nivel preuniversitario y cotejo con tres fases de adquisición). Turku: Universidad de Turku.

Dimitrijević, N. (1969): Lexical Availability. Heilderberg: Julius Groos Verlag. Flavell, J. H. (1985): Cognitive Development. Englewood Cliffs, N.J: Prentice- Hall.

Gerstl, P., Pribbenow, S. (1995): «Midwinters, end games, and body parts: a classification of part-whole relations». Journal of Human and Computer Studies, 43, 865-889.

Ibbotson, P., Tomasello, M. (2009): «Prototype constructions in early language acquisition». Language and Cognition, 1, 59-85. 
Hernández Muñoz, N. (2006): Hacia una teoría cognitiva integrada de la disponibilidad léxica. El léxico disponible de los estudiantes Castellano-manchegos. Salamanca: Universidad de Salamanca.

Iglesias, J.L. (2015): «¿Cómo influye la moda en el comportamiento de los adolescentes?». Adolescere, III(1), 57-66.

Jiménez Catalán, R., Fernández Fontecha, A. (2019): «Lexical Availability Output in $\mathrm{L}_{2}$ and $\mathrm{L}_{3}$ EFL learners. Is There a Difference?». English Language Teaching, 12(2), 77-89.

Jiménez Catalán, R., Agustín Llach, M.P. (2017): «CLIL or time? Lexical profiles of CLIL and non-CLIL EFL learners». System, 66, 87-99.

Jiménez Catalán, R. (ed.) (2014): Lexical Availability in English and Spanish as a Second Language. Dordrecht/Heidelberg/London/New York: Springer.

Kleiber, G. (1995): La Semántica de los Prototipos. Madrid: Visor.

Lakoff, G. (1987): Women, Fire, and Dangerous Things. What Categories Reveal about the Mind. Chicago: The University of Chicago Press.

Lakoff, G., M. Johnson. (1980): Metáforas de la vida cotidiana. Madrid: Cátedra. Lakoff, G., M. Johnson. (1999): Philosophy in the flesh. New York: Basic Books.

Lakoff, G., M. Turner. (1989): More than cool reason. Chicago: University of Chicago Press.

Larrochelle, S., H. Pineau (1994): «Determinants of response times in the semantic verification task». Journal of Memory and Language, 33, 796-823.

López Chávez, J. (1995): Léxico fundamental panbispánico: realidad o utopia. En: Actas del III Congreso Internacional sobre el español de América. Santiago de Chile: Universidad Católica de Chile, 1006-1014.

López González, A. (2014): «Desarrollo de los estudios de disponibilidad léxica en Español Lengua Extranjera (ELE)». En: Narciso M. Contreras Izquierdo (ed.), La enseñanza del español como LE/L2 en el siglo XXI. Málaga: ASELE, 397-408.

Lukusa, S. (1996): «A semantic prototype of the English word "responsible" in Tanzanian usage». UTAFITI (New Series), 3(1), 145-160.

Murphy, G (2002): The big book of concepts. Cambridge, Massachusetts, London: The MIT Press.

Orduz, L.F. (2010): «Púberes, adolescentes, jóvenes». Conferencia ofrecida en el VI Encuentro de Bienestares Universitarios de las Universidades Católicas y de Inspiración Cristiana. Bogotá. 
Paredes García, F. (2015): «Disponibilidad léxica y enseñanza de ELE: el léxico disponible como fuente curricular y como recurso en el aula». Linred: Lingüistica en la Red, 13, 2015-16.

Piaget, J., Inhelder, B. (1969): The Psychology of the child. Michigan: Basic Books.

Rosch, E (1975): «Cognitive Representations of semantic categories». Journal of Experimental Psychology. General, 104, 192-233

Rosch, E., Lloyd, B. (1973): Cognition and Categorization. Hillsdale, New Jersey: Lawrence Erlbaum Associates.

Rosch, E., Mervis, C. (1975): «Family Resemblances: Studies in the internal structure of categories». Cognitive Psychology, 7, 573- 605

Samper Hernández, M. (2002): Disponibilidad léxica en alumnos de español como lengua extranjera. Málaga: ASELE. Colección Monografías, 4.

Samper Padilla, J. A. (1999): «Léxico disponible y variación dialectal: datos de Puerto Rico y Gran Canaria». Estudios de lingüística bispánica: bomenaje a María Vaquero. San Juan de Puerto Rico: Universidad de Puerto Rico, 550-573.

Scalozub, L.T. (2007): «El protagonismo del cuerpo en la adolescencia». Psicoanálisis, 29(2), 377-391.

Supasiraprapa, S. (2017): «Prototype Effects in First and Second Language Learners: The case of English Transitive Semantics». Bilingualism, Language and Cognition, 21(3), 618-639.

Šifrar Kalan, M. (2009): «Disponibilidad léxica en español como lengua extranjera: el cotejo de las investigaciones en Eslovenia, Salamanca y Finlandia». Verba Hispánica, 17, 165-182.

Šifrar Kalan, M. (2016): «La universalidad de los prototipos semánticos en el léxico disponible de español». Verba Hispánica, 24, 147-165.

Vigotsky, L. (1995): Pensamiento y Lenguaje. Barcelona: Paidós

Warrington E. K., R. A. McCarthy (1987): «Categories of knowledge». Brain, 110, 1273-1296.

Yuan, R. (1990): «Semantic Transferability and Prototypicality in Chinese and English: a study of the semantic acquisition of "thin" by Chinese learners of English». Retrospective Theses and Dissertations 86: https: // lib.dr.iastate. $\mathrm{edu} / \mathrm{rtd} / 86$ (7-11-2019). 
Jaqueline Mora

Rosa Ma Jiménez Catalán

University of Rioja

\section{Prototypical associations in the production and lexical availability of Spanish and Slovene adolescents in Spanish and in English as foreign languages}

Keywords: prototypes, word associations, productive vocabulary, lexical availability, parts of the body, clothes, foreign language, Spanish, English

Semantic prototypes allow us to understand the categorization of a language. This study on prototypes in two foreign languages with informants from two different countries enables us to address the prototypical associations in a global framework. This study compares a group of 100 Slovenian students of secondary education learning Spanish as a foreign language, and a group of 100 Spanish students learning English as a foreign language. We intend to examine two categories, parts of the body and clothes, with the following objectives: a) to determine if similarities or differences are observed in the number of words produced by Spanish and Slovenian adolescent learners of English and Spanish as foreign languages, respectively, in the categories of parts of the body and clothes; and b) to ascertain if similarities and/or differences are observed in the most available words for those categories in English and Spanish. Specifically, we aim to determine whether there are either convergences or divergences in prototypes, both in English and Spanish. Our results suggest the existence of shared prototypes within the two semantic categories analysed in English and Spanish. Moreover, although to a lesser extent, our results also point to the existence of cultural idiosyncrasies in clothes/la ropa. 
Jaqueline Mora

Rosa Ma Jiménez Catalán

Univerza v La Rioji

\section{Prototipne asociacije $\mathbf{v}$ produkciji besedišča in leksikalni razpoložljivosti pri španskih in slovenskih najstnikih $\mathbf{v}$ španščini in angleščini kot tujih jezikih}

Ključne besede: prototipi, besedne asociacije, produktivno besedišče, leksikalna razpoložljivost, deli telesa, oblačila, tuji jezik, španščina, angleščina

Semantični prototipi omogočajo razumevanje kategorizacije jezika. Na osnovi študije teh prototipov $\mathrm{v}$ dveh tujih jezikih $\mathrm{z}$ informanti iz dveh različnih držav lahko prototipne asociacije obravnavamo s celostnega vidika. V študiji primerjamo skupino stotih slovenskih dijakov, ki se španščine učijo v Sloveniji, in skupino stotih španskih učencev angleščine v okviru srednješolskega izobraževanja v Španiji. Analizirali smo zmožnosti obeh skupin na dveh semantičnih poljih. Naš namen je (1) določiti, ali gre opaziti podobnosti ali razlike $\mathrm{v}$ številu produciranih besed med španskimi in slovenskimi najstniki v angleščini in španščini kot tujih jezikih na poljih Deli telesa in Oblačila, in (2) raziskati, ali obstajajo podobnosti in/ali razlike na področju najbolj razpoložljivih besed $\mathrm{z}$ danih polj $\mathrm{v}$ angleščini in španščini. Rezultati kažejo na obstoj skupnih prototipov $\mathrm{v}$ angleščini in španščini na analiziranih poljih. Kljub temu pa, čeprav v manjši meri, rezultati nakazujejo tudi na prisotnost kulturno pogojenih posebnosti na polju Oblačila. 